\section{TRANSCATHETER AORTIC VALVE IMPLANTATION AND ADAPTIVE / PROGRESSIVE COVERAGE}

doi:10.1017/50266462314000312

\section{PRESENTATION OF GENERIC TAVI-CASE: BACKGROUND}

Transcatheter aortic valve implantation (TAVI or TAVR) is a newly introduced medical procedure enabled by a new device. Although its introduction does not represent that of all medical devices, it does illustrate a situation in which a device provides a new alternative to standard care in a specific group of patients. Unlike drugs, the medical device category is highly heterogeneous and includes both diagnostic and treatment (accessories, implantable, enablers) and capital equipment. The TAVI- case was discussed as one of the examples in the Policy Forum Meeting and the authors were acting as facilitators that summed up both the case as presented and the discussion between the members that followed. We wish to give a special thanks to the participants of the group work for their input to the discussion.

Several different products for TAVI have been introduced by multiple producers (Edwards LifeScience; Medtronic; Symetis; JenaValve; St. Jude Medical Inc; Direct Flow Medical; others). Regulatory approval has not created substantial barriers to patient access either in the United States or in the European Union. Both regulatory regimes have sequentially approved new market licenses based on new data which demonstrate that the newer version performs equal to or better than the older version or when new clinical data supporting use in broader populations has become available.

Decisions regarding coverage have created a larger barrier to access. These decisions have reflected two related issues: First, because the TAVI procedure is a new one (percutaneous procedure), in countries where hospital care is covered by a DRG system, it has required the creation of new codes; Second, there has been substantial uncertainty regarding real world performance and the clinical and cost-effectiveness that can be achieved with its introduction. Like other new procedures, use of the device requires appropriate training of a medical team who will select appropriate patients and implant the devices.

Adaptive approaches to access in this example have the potential to reduce payer uncertainty about real-world performance, as well as providing information about potential safety issues or key subgroups of patients in which the benefit and cost-effectiveness of the therapy is maximised.

\section{RESULTS FROM GROUP DISCUSSIONS}

Adaptive processes, whether during the regulatory process or at the reimbursement level, can be desirable under great uncertainty or in situations where one may need to accommodate for regular or rapid changes in technology over time. This is typically the case with many medical devices and procedures. Adaptive processes might also be desirable for drugs and technologies with high expected value and in disease groups with no beneficial treatment alternatives. Adaptive processes might reduce the length of development time for industry and at the same time provide early access to selected groups of patients. Patient access can be broadened over time if the target population/indications progressively adapt, as new evidence becomes available. Such a process requires better coordination of regulatory and reimbursement decisions alongside further data collection.

Creating adaptive pathways should involve all stakeholders at all stages and be flexible or have multiple paths to accommodate a variety of situations. They also have to be transparent and predictable, but not too rigid. The intent is to lower uncertainty for all stakeholders by incorporating new data as evidence emerges. This approach would enable getting data earlier which could lead to a more manageable risk which may be formal or informal (system may adapt without rules). 
Obvious benefits to the system would be that information and feedback from clinicians and patients to innovators could be collected more systematically and would allow for accommodating the learning curve inherent to any procedure. Relevant and updated information could be given to patients and adaptive processes would prevent overuse of technologies before evidence is good enough.

On the other hand, adaptive approaches are demanding for all parties involved and it is anticipated that the coordination and management of such pathways might require more resources than the current processes. Remaining questions regarding who will fund the processes are still open and political aspects cannot be avoided, however having a clear process, with a set of criteria preliminarily agreed upon by the stakeholders should continue to increase the transparency and minimize issues.

One key aspect is the definition of criteria in cases of unmet need, when "high value" is expected and there is no good existing gold standard. In these cases, the criteria might be linked to the disease (type and severity), the targeted patient population(s), and the harm/benefit balance.

Given the diversity of medical devices, there is also a need to define for which type of medical devices an adaptive approach could be applied. Depending on the situation, the goal could be either to maximize the benefit of a new technology or to minimize the risks.

A key challenge is the differences in processes between legislations / countries. Having adaptive coverage in place would be a departure from the TAVI example where patient access greatly differs from one country to the other. It would mean having a coordinated system allowing patient access with data collection along the way to broaden/refine the target population. With time the technology itself would be expected to change (i.e., modifications to the device) as well as the procedural aspects such as expanding to trans-apical and femoral access.

Finally, TAVIs illustrate the need to address the clear disconnect between regulatory/reimbursement pathways and purchasing processes for in-hospital medical devices. Adaptive processes would require better coordination, and ideally upstream of technology introduction.

\section{CONCLUSIONS}

The process of adaptive pathways, whether to facilitate licensing or coverage, needs to be transparent, flexible, and predictable. One key benefit would be to bring to industry more clarity on the requirements linked to market access in general and as a consequence better predictability for research and development decisions. An adaptive approach will also have an impact on organizational, economic, social, and ethical aspects.

\section{Marianne Klemp}

Norwegian Knowledge Center for the Health Services, Oslo, Norway

\author{
Sophie Cros \\ Abbott Vascular International, Diegem, Belgium \\ Mitchell Sugarman, on behalf of the HTAi Policy Forum \\ Medtronic, Santa Rosa, 95403 California, USA
}

\section{HTAI POLICY FORUM: KEEPING HTA ON TRACK}

do: $10.1017 / 50266462314000336$

The most recent discussions of the HTAi Policy Forum are being showcased in this issue of our Journal. The topic of adaptive approaches to technology management has been a policy area of continuing importance and relevance to health technology assessment agencies and policy makers as well as industry. The latest Policy Forum publication (Husereau et al., in this issue) demonstrates that the discussions at the meeting about this complex topic were very wide indeed.

Our HTAi Policy Forum is unique in providing the opportunity for senior people from organizations in the public and private sector to discuss HTA topics of strategic importance in a safe and nurturing environment. Discussions during Policy Forum meetings usually reveal a wide range of views. Indeed a key attribute of the Forum is that it manages to make participants feel sufficiently safe that they air their genuine perspectives, having been informed by excellent briefing papers and expert commentaries. In this way, the Forum manages to identify fundamental issues associated with the topic, highlight where viewpoints align and differ and provide commentary on key actions needed to make progress.

From its inception, the Policy Forum has not shied away from tackling difficult topics. The publications developed from Policy Forum discussions (1-8) are well worth reading and freely accessible on the HTAi website. Looking back at this output since 2007 gives an indication that "technology management" has been, in some shape or form, an area of interest for most meetings. Policy Forum discussions about coverage with evidence development, managed entry arrangements, optimal use of technologies and interaction between HTA bodies and regulators are, of course, interrelated to each other and closely linked to the current topic. Whilst this might suggest a rather narrow scope of interests for Policy Forum members, it is quite the opposite. We cannot overestimate the importance of constructing an interdependent, mutually beneficial ecosystem between life science $\mathrm{R}+\mathrm{D}$ and healthcare systems, to deliver on behalf of the public at large. This, coupled with the complexity involved in contemplating how current systems need to develop, means that it is necessary to spend time exploring the issues from many angles and with a variety of viewpoints. 